\title{
gs \\ Atomic configuration and mechanical and electrical properties of stable gold wires of single-atom width
}

\author{
Tokushi Kizuka* \\ Institute of Materials Science, University of Tsukuba, Tsukuba, Ibaraki 305-8573, Japan \\ (Received 14 December 2007; revised manuscript received 28 January 2008; published 1 April 2008)
}

\begin{abstract}
The formation dynamics of stable gold $(\mathrm{Au})$ wires of single-atom width during tensile deformation of the nanometer-sized $\mathrm{Au}$ contacts was observed in situ at room temperature by transmission electron microscopy. Simultaneously, force and conductance were measured using functions of scanning probe microscopy. The wires were extended to $2.7 \pm 0.1 \mathrm{~nm}$ and the number of atoms was increased to 10 ; the average interatomic distance was $0.30 \pm 0.02 \mathrm{~nm}$. The duration of the observation of the wires exceeded $10 \mathrm{~s}$. The results of the simultaneous observation of the atomic configuration, force, and conductance led to the conclusion that the stable Au wires observed in this study are complexed with light-element atoms. The constants of the mechanical properties of the individual wires, i.e., elastic limit, Young's modulus, and strength, were analyzed on the basis of stress-strain curves experimentally measured on an atomic scale. During elastic elongation, a force of $1.6 \pm 0.7 \mathrm{nN}$ acted on the Au wires with an elastic limit of 0.15-0.20. Young's modulus of single-atom-width Au wires was 47-116 GPa and strength was 8-17 GPa, estimated using a cross-sectional area of $0.118 \mathrm{~nm}^{2}$, which is that of a single atom in bulk along $\langle 110\rangle$. Using these mechanical constants, the average interatomic distance in stress-free wires was estimated to be $0.26 \pm 0.02 \mathrm{~nm}$. The histogram of conductances for the single-atom-width Au wires showed a peak at approximately $1 G_{0}$ (where $G_{0}=2 e^{2} / h, e$ being the charge of an electron and $h$ Planck's constant) until the total number of atoms exceeds 5. For longer wires constructed of more than six atoms, the conductance decreased to less than $0.1 G_{0}$.
\end{abstract}

DOI: 10.1103/PhysRevB.77.155401

PACS number(s): 61.46.Km, 68.37.Og, 62.23.Hj, 68.65.La

\section{INTRODUCTION}

Atomic-sized wires (ASWs), i.e., one-dimensional arrays of single atoms, are the smallest possible structures of solid contacts and are produced by retraction of nanometer-sized contacts (NCs). ${ }^{1-14} \mathrm{NCs}$ composed of metallic elements are studied in relation to their quantized conductance defined by a quantum $\left(G_{0}=2 e^{2} / h\right.$, where $e$ is the electron charge and $h$ is Planck's constant). ${ }^{15-29}$ For gold (Au) NCs, the conductance decreases in a stepwise variation during the thinning process, and the conductance level at each step corresponds to approximately integer multiples of $G_{0}{ }^{2,3,5-7}$ According to Landauer formula, for a conductance level of approximately $1 G_{0}$, the cross-sectional area of the contact is estimated to be that of a single gold atom. ${ }^{2,3,5,26,27}$ Molecular dynamics and $a b$ initio calculations have shown a similar relationship between the conductance and the cross-sectional area. ${ }^{13,30-35}$ It is pointed out, on the basis of theoretical analysis and tightbinding and free-electron calculations, that the conductance of Au NCs and ASWs are sensitive to irregularities in the wire shape, decrease in the convergent angle of electrodes, and small variation in the interatomic distance. ${ }^{18,25,36,37}$ Thus, the quantized conductance is destroyed owing to this sensitivity. Recently, a classical molecular dynamics simulation coupled with conductance calculations based on a tight binding model shows that the conductance of the single-atom $\mathrm{Au}$ contacts ranges from $0.5 G_{0}$ to $2.0 G_{0} \cdot{ }^{38}$ According to $a b$ initio calculations, the conductance of pure Au ASWs decreases from $1 G_{0}$ owing to expansion and dimerization. ${ }^{33,35,39,40}$ However, the interatomic distances of Au ASWs showing a conductance of $1 G_{0}$, measured by mechanically controllable break junction techniques (MCBJTs) and nanotip contact techniques by scanning tunneling microscopy (STM) and high-resolution transmission electron microscopy (HRTEM), range widely from 0.25 to $0.40 \mathrm{~nm} \cdot{ }^{9,14,41-45}$ In addition, the conductance of ASWs is affected by the incorporation of light-element atoms in ASWs and the adsorption of gaseous molecules on ASWs. ${ }^{34,35,46-51}$ HRTEM enables us to observe directly the atomic configuration of ASWs. ${ }^{28,41-45,52-67}$ However, ASWs observed by HRTEM have been restricted to less than five constituent atoms owing to instability. Therefore, the atomic configuration of longer stable ASWs and the relationship to their electrical and mechanical properties still remain open problems.

In this study, we directly observed the variation in atomic configuration during the formation of $\mathrm{Au} \mathrm{ASWs}$ and the elongation process to ten atoms in length for up to $10 \mathrm{~s}$ by the in situ HRTEM combined with conductance and force measurements. From the observation of the structural dynamics on an atomic scale, we derive stress-strain relations and the relationship between the atomic configuration and the conductance. As a result, we demonstrate experimental evidence for the relationships among the atomic configuration and mechanical and electrical properties of the long $\mathrm{Au}$ ASWs.

\section{EXPERIMENTAL METHODS}

The experimental method in this study was developed on the basis of in situ HRTEM combined with subnanonewton force measurements used in atomic force microscopy (AFM) and electronic conductance measurements used in scanning tunneling microscopy. ${ }^{52,53,58,61}$ First, we prepared nanometersized Au tips: Au was evaporated in a vacuum chamber and deposited on a Si cantilever with a nanotip. The cantilever tip 
was attached to the front of a piezo tube on a cantilever holder for HRTEM. A Au plate of $0.2 \mathrm{~mm}$ thickness was attached to the second plate holder. The contact edge of the plate for contact was thinned to $5-20 \mathrm{~nm}$ by argon ion milling. The cantilever and plate holders were then inserted into the in situ transmission electron microscope at the University of Tsukuba. The specimen chamber of the microscope was evacuated first by a turbomolecular pump and then by an ion pump, resulting in a vacuum of $1 \times 10^{-5} \mathrm{~Pa}$ with typical residual gaseous molecules of $\mathrm{H}_{2}, \mathrm{O}_{2}, \mathrm{H}_{2} \mathrm{O}, \mathrm{CO}, \mathrm{CO}_{2}, \mathrm{~N}_{2}$, and hydrocarbon. The cantilever tip was brought into contact with the edge surface of the opposing plate by piezomanipulation while applying a bias voltage of $13 \mathrm{mV}$ between the tip and the plate. The cantilever tip was then pressed into the plate to prepare NCs and then retracted to elongate them. A series of these manipulations were performed at room temperature. The structural dynamics during the process was observed in situ by lattice imaging by HRTEM using a television capture system. The time resolution of image observations was $17 \mathrm{~ms}$. The force applied between the tip and the plate was simultaneously measured by the optical detection of cantilever deflection. The electrical conductance was measured using a two-terminal method. The results of high-resolution imaging and signal detection in this system were simultaneously recorded and analyzed for every image. Image simulation for HRTEM observation of ASWs was performed using Ishizuka's multislice method under the Scherzer focus condition with $256 \times 256$ waves. ${ }^{68}$

\section{RESULTS}

Figure 1 shows a time-sequence series of high-resolution images of the formation process of an ASW during the tensile deformation of a Au NC. The Au tip and plate are observed as dark areas in the upper and lower parts of Figs. $1(a)-1(1)$. The plate is negatively biased by $13 \mathrm{mV}$ to the tip. In the middle of Fig. 1(a), the contact of approximately $1 \mathrm{~nm}$ width between the tip and the plate is observed. On the surfaces of both the tip and the plate, neither contamination nor an oxide layer is observed throughout Figs. 1(a)-1(1). As the tip is retracted from the plate along the direction indicated by the arrow in Fig. 1(a), the NC is thinned, as shown in Figs. 1(a)-1(d). Then, the contact width decreases to the length of a single atom, i.e., an ASW of five atoms in length forms, as shown in Fig. 1(e). At such transformation from a NC to an ASW, the stress on ASWs increases rapidly owing to an abrupt decrease in the cross-sectional area. As a result, the tip speed increases and the images blur during the transformation. In Fig. 1, we thereby show the ASW of five atoms after blurring as the start of structural analysis. The tip speed depends on the retraction speed of the tip and dose not necessariliy imply lower stability of ASWs of shorter than four atoms in length. The ASW grows, as shown in Figs. 1(e) $-1(\mathrm{k})$, and finally breaks, as shown in Fig. 1(1). During the growth, the shape of both the tip and the plate varies and the connecting point between the plate and the ASW shifts to the left-hand side of each frame. The variation in the tip and plate shape is not caused by electron beam sputtering since no damage due to the electron beam was observed on either the tip or the plate after fracture. Thus, this observation shows that the growth of the ASW occurs owing to the extraction of atoms from both the tip and the plate. As reported by Fujii et al. ${ }^{69}$ and Smit et al., ${ }^{70}$ current-induced force acting on atoms in Au NCs increases remarkably at a bias voltage of more than $1.0 \mathrm{~V}$. The bias voltage used in this study was $13 \mathrm{mV}$, much lower the critical voltage, and typical electromigration from negative electrode to positive electrode ${ }^{71}$ was not dominant. The formation probability of stable ASWs per retraction process, which could be confirmed by the HRTEM, as shown in Fig. 1, was estimated to be less than $5 \%$. This low probability is consistent with the results obtained by STM. ${ }^{72,73}$

Since this growth occurs during retraction, a tensile force acts on the contact in Fig. 1. After the tip and the plate were separated, no damage due to the electron beam was observed on either the tip or the plate. Thus, this formation process of ASW by retraction is in contrast to that by intense electron beam sputtering, by which two neighboring holes are produced in a metallic film by knock-on of the constituent atoms with high-energy electrons and a wire structure emerges between these two holes. ${ }^{41,43-45,57}$ In the electron beam sputtering method, the constituent atoms of the wire are desorbed into a vacuum, and a controlled force is not loaded. On the other hand, during the present formation process of the ASW by retraction with a tensile force, the number of atoms of the ASW and the two connecting electrodes is conserved. Therefore, the resultant stable ASW structures produced by these methods are not necessarily equal owing to the different formation conditions. In the present method, the formation process of the ASW is governed by atomistic dynamics under a tensile condition.

Figure 2 shows a simulation result for an HRTEM image of a Au ASW. The model for the simulation is composed of a wire part of ten $\mathrm{Au}$ atoms and two pyramidal Au crystals connected at the two ends of the wire part, as shown in Fig. 2(a). The ten $\mathrm{Au}$ atoms are aligned along the [001] direction of the pyramidal crystals with an interatomic distance of $0.288 \mathrm{~nm}$, which is that of coarse-grained Au crystals, as shown in Fig. 2(b). Figure 2(c) is the simulated image corresponding to the model. The intensity profile along the centerline of the ASW $\left[P_{s}-P_{e}\right.$ in Fig. 2(c)] is shown in Fig. 2(d). From the comparison of the simulated to the model, we find that the Au atoms of the ASW appear as black dots in the intensity image. Furthermore, the center of the atoms corresponds to that of the black dots within a deviation of $0-0.02 \mathrm{~nm}$. The maximum deviation is observed at the second atom from either end. The darkest positions for the images of the second atoms shift by $0.01-0.02 \mathrm{~nm}$ toward the central point of the ASW because of the bright Fresnel fringes caused by the thick pyramidal crystals at either end, which overlap with the image of the ASW. Since the spatial resolution of the present observation is $0.1 \mathrm{~nm}$, the amount of shift is not detected in this study. This simulation result also reveals that, to preclude this overlap effect, the ASWs constructed of at least five atoms should be used to estimate accurately the position of the atoms from TEM images.

Figure 3 shows the extraction of the ASW image from Fig. 1(k) and its intensity profile along the centerline, similar to those given in Fig. 2. The darkest positions are indicated 

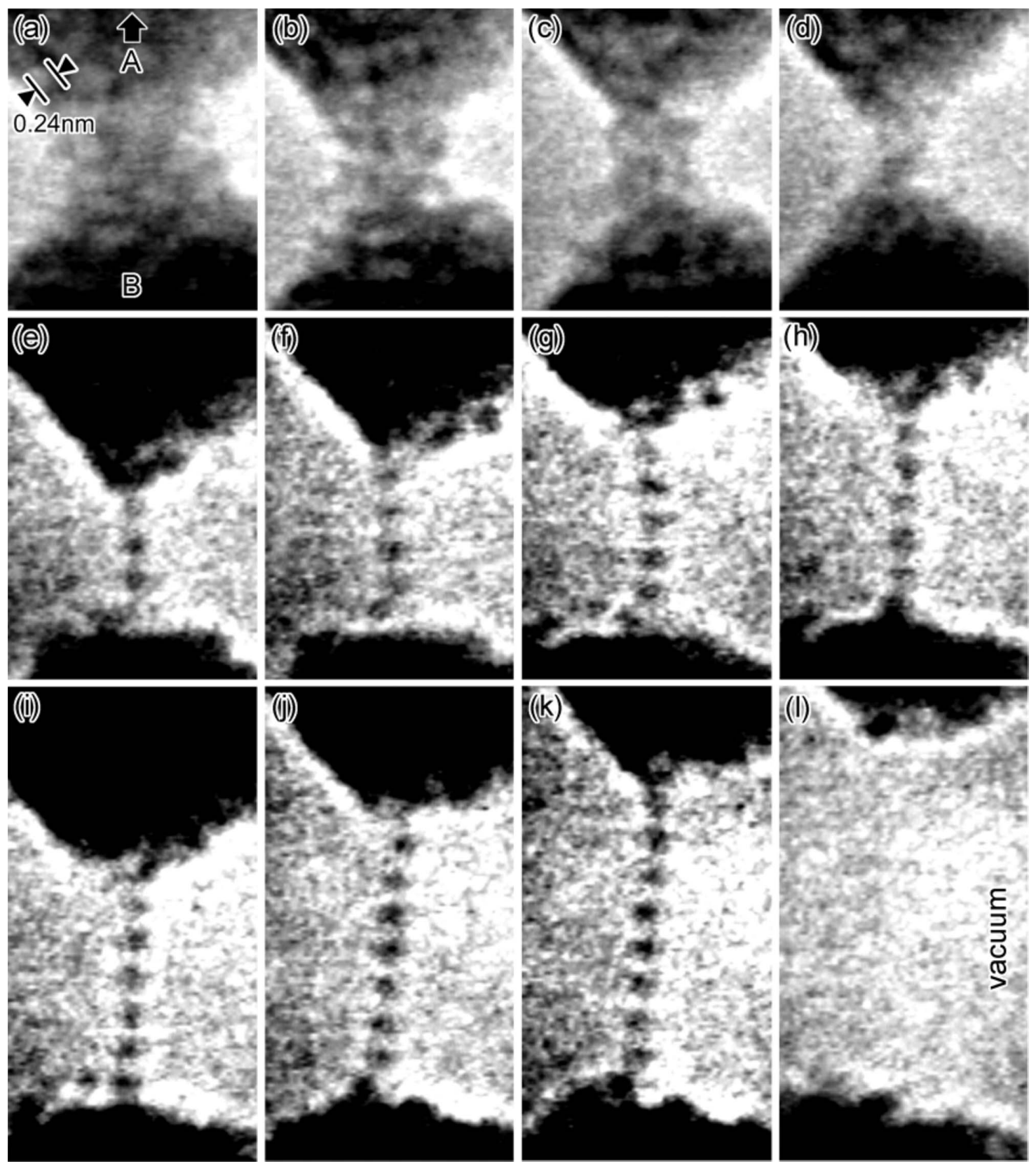

FIG. 1. Time-sequence series of high-resolution images of formation process of ASW during tensile deformation of Au NC. The NC is observed between (A) the tip and (B) the plate. The spacing of (111), i.e., $0.24 \mathrm{~nm}$, is shown in (a). The brighter area is the vacuum. The contrast in (e)-(1) is increased to show the ASW clearly. A voltage of $13 \mathrm{mV}$ was applied between the tip and the plate. The tip was retracted from the plate along the direction indicated by the arrow in (a).

by arrows. In accordance with the simulation result, the atom positions of the ASW shown in Figs. 1(e)-1(k) are concluded to be at the darkest positions in the profile. The sequence of those positions is illustrated in Figs. 4(e)-4(k). The ASW in Figs. 4(e) $-4(\mathrm{~g})$ arches slightly even during retraction, whereas the ASWs in Figs. 4(h)-4(k) are almost straight.

Figure 5 shows variations in the separation distance between the tip and the plate observed in Fig. $1(L)$, the total length of the ASW $(\ell)$, the number of atoms in the ASW $(N)$, the interatomic distance within the ASW $(d)$, and the conductance of the contact during tensile deformation $(G)$ as a function of time. The times indicated by arrowheads a to 1 in
Fig. 5 [hereafter, times (e)-(k)] represent the times at which the images in Figs. 1(a)-1(1) were observed. The inclination of the separation distance, i.e., the retraction speed, was controlled at $0.077 \mathrm{~nm} / \mathrm{s}$ in time region $(\mathrm{e})-(\mathrm{g})$ and increased to $0.19 \mathrm{~nm} / \mathrm{s}$ in time region $(\mathrm{h})-(\mathrm{k})$. As the separation distance increases, the total length increases. The number of atoms also increases from five at time (e) to ten at time (k). The duration through which the ASW maintains the same number of atoms is $1-3 \mathrm{~s}$, indicating that the ASW is sufficiently stable to observe its structure by HRTEM. On the other hand, the average interatomic distance remains $0.27-0.32 \mathrm{~nm}$ throughout this growth. The conductance of the contact de- 
(a) $\bar{Q}$

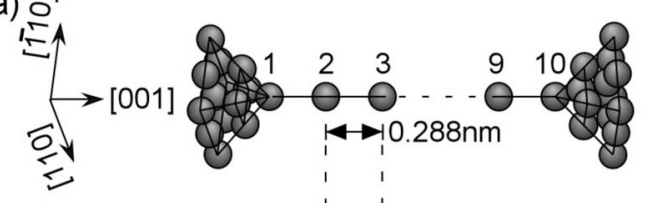

(b)
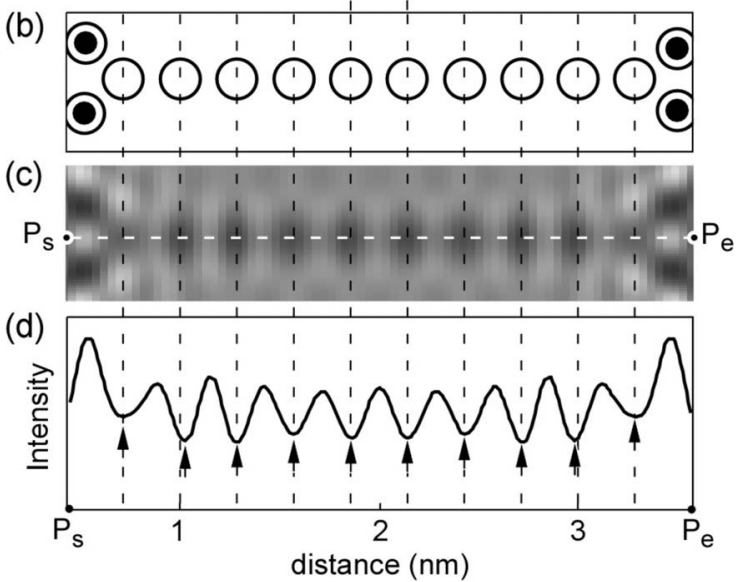

FIG. 2. Simulation of high-resolution image of Au ASW. (a) Bird's eye view of model of atom configuration of ASW and connecting electrodes and (b) projected view of model along electron beam direction ([110]). The interatomic distance of the ASW was selected to be $0.288 \mathrm{~nm}$, which corresponds to the nearest-neighbor distance in bulk Au. (c) Result of simulation for model in (b). (d) Line profile of intensity along the centerline of ASW, i.e., $P_{s}-P_{e}$ in (c). The broken lines and the arrows in (d) show the positions of atoms and the local minima of the intensity, respectively.

creases to $\sim 1 G_{0}$ after the ASW forms at time (e). We show the conductance variation with an enlarged view in Fig. 6(a). It is noted that, even during the time region with the same number of atoms, the conductance curve shows a discrete up-and-down variation between $0.2 G_{0}$ and $1.5 G_{0}$, as observed from time (d) to time (e). This reveals that, after the increase in the number of atoms, structural relaxation results in the recovery of conductance. In Fig. 6(b), we show the histogram of the count at each conductance value. A peak is observed at approximately $1 G_{0}$ and the corresponding plateaus appear for $\sim 1 \mathrm{~s}$ from time (e) to time (f). In this time region, the number of atoms is 5. Therefore, this ASW constructed of five atoms has at least several quasi-stable atomic

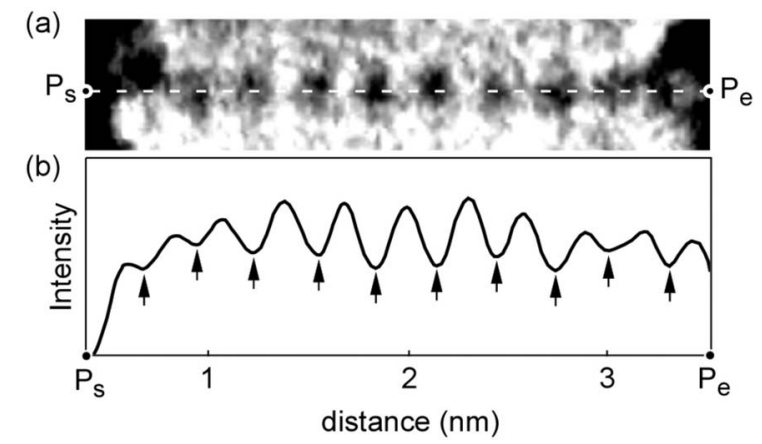

FIG. 3. (a) High-resolution image of ASW in Fig. 1(k) and (b) its line profile of intensity along $P_{s}-P_{e}$ in (a). The arrows in (b) indicate the local minima of the intensity.

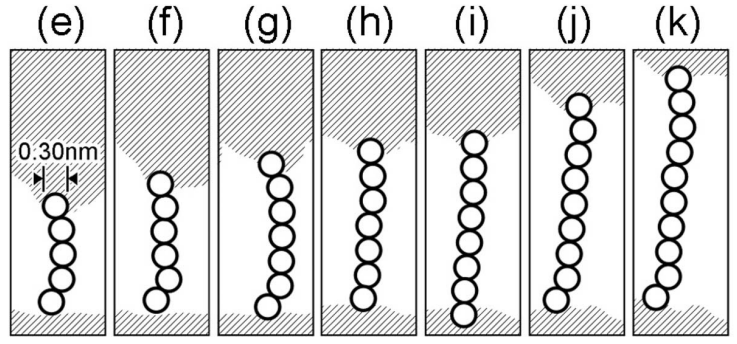

FIG. 4. Configurations of atoms in ASW in (e)-(k) correspond to Figs. 1(e)-1(k), respectively. The diameter of the circles is selected to be the average interatomic distance of the ASW observed in Fig. 1.

configurations. After time (g), when the number of atoms exceeds 5 , the conductance further decreases, to values less than $0.1 G_{0}$.

The histogram shown in Fig. 6(b) is calculated from the retraction process shown in Fig. 1. To investigate general conductance features observed by this in situ HRTEM, we selected 40 retraction processes in which NCs were gradually thinned to ASWs, and then constructed a conductance histogram, as shown in Fig. 7. Peaks are observed at integer multiples of $G_{0}$, which is a well-known feature of the conductance of Au NCs. ${ }^{7,8,29}$ It is also noted that, at $0.5 G_{0}$, a peak appears. The HRTEM observation showed that the $0.5 G_{0}$

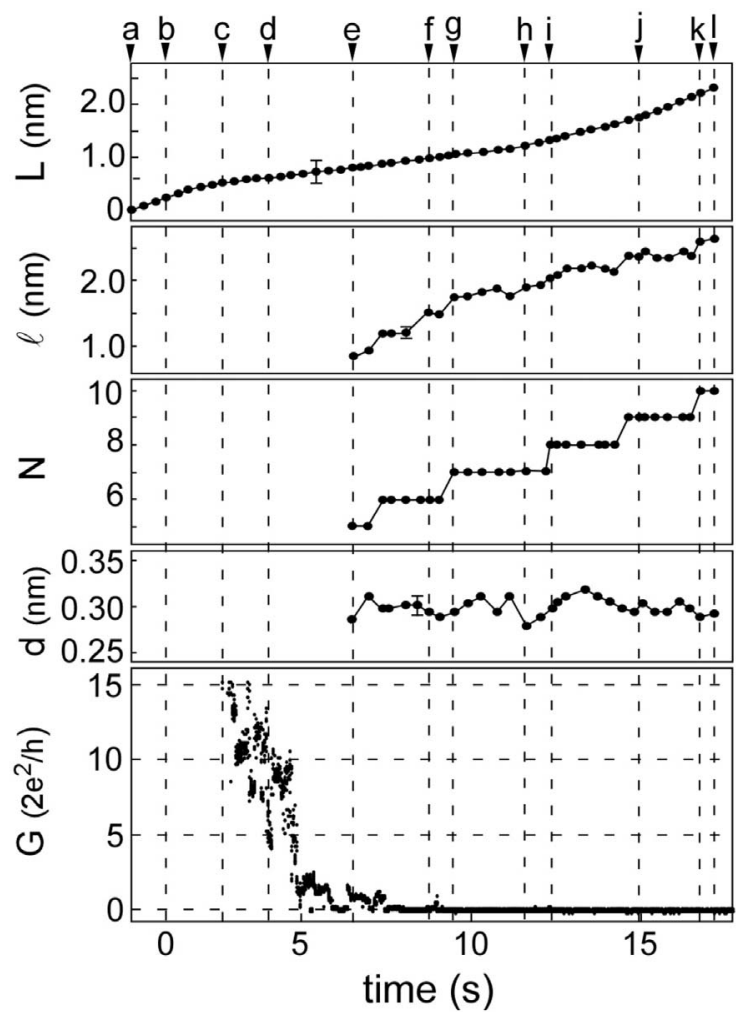

FIG. 5. Variations in separation distance of tip and plate observed in Fig. $1(L)$, total length of ASW observed in Figs. $1(\mathrm{e})-1(\mathrm{k})(\ell)$, number of atoms in $\operatorname{ASW}(N)$, interatomic distance in ASW $(d)$, and conductance of contact during the tensile deformation $(G)$ as function of time. The images in Figs. 1(a)-1(1) were observed at the times indicated by arrowheads (a)-(1). 


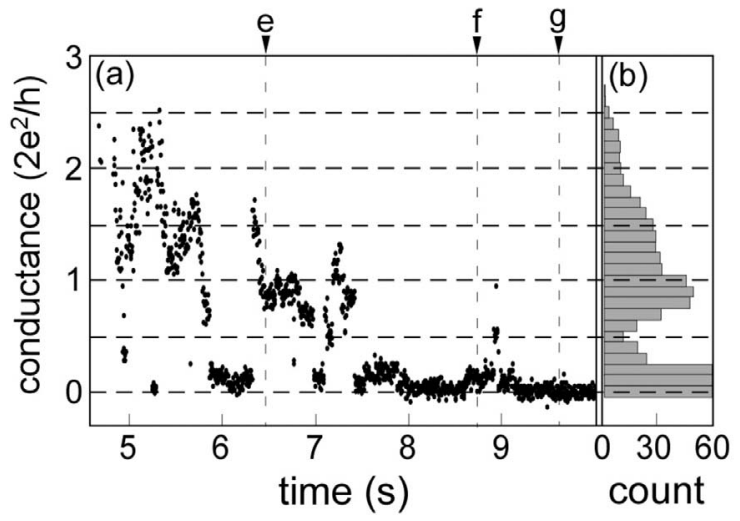

FIG. 6. (a) Enlarged view of conductance in Fig. 5 and (b) histogram of conductance values in this time region. Times indicated by arrowheads (e)-(g) corresponds to times (e)-(g) in Fig. 5.

peak is related to the count accumulation from ASWs, as similarly seen in Fig. 6(b). According to the previous reports, to resolve peaks at quantized levels, several thousand ran-

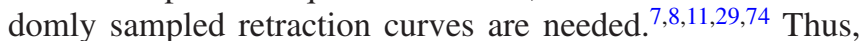
our result of histogram construction reveals that the conductance during the gradually thinning process contributes to peak sharpening at the quantized levels.

Figure 8 shows histograms of the average interatomic distances measured for ASWs constructed of five to ten atoms. The mode for each number of atoms is distributed from 0.28 to $0.33 \mathrm{~nm}$. The average interatomic distances for each number of atoms are also plotted on the right side of Fig. 8. The average interatomic distances of ASWs range from 0.25 to $0.35 \mathrm{~nm}$. The minimum and maximum interatomic distances measured in an ASW are $0.25 \pm 0.03$ and 0.40 $\pm 0.03 \mathrm{~nm}$, respectively. The average value of the total interatomic distances is $0.30 \pm 0.02 \mathrm{~nm}$. This feature is also seen in Fig. 5. To demonstrate the difference in the structure of the observed ASWs having the average, minimum, and maximum interatomic distances, i.e., $0.30 \pm 0.01,0.25 \pm 0.02$, and $0.40 \pm 0.03 \mathrm{~nm}$, respectively, we show their high-resolution images in Figs. 9(a)-9(c). As observed in Figs. 9(a)-9(c), interatomic distances in an ASW are not homogeneous. In particular, the atomic configurations seen in Figs. 9(b) and 9(c) are comparable to the configuration of dimer-coupled

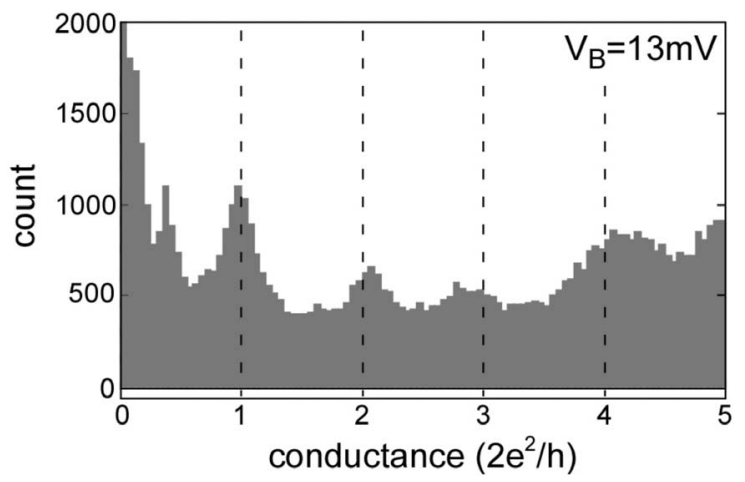

FIG. 7. Histogram of conductance values measured during retraction process of $40 \mathrm{Au}$ NCs biased by $13 \mathrm{mV}$ between tip and plate.

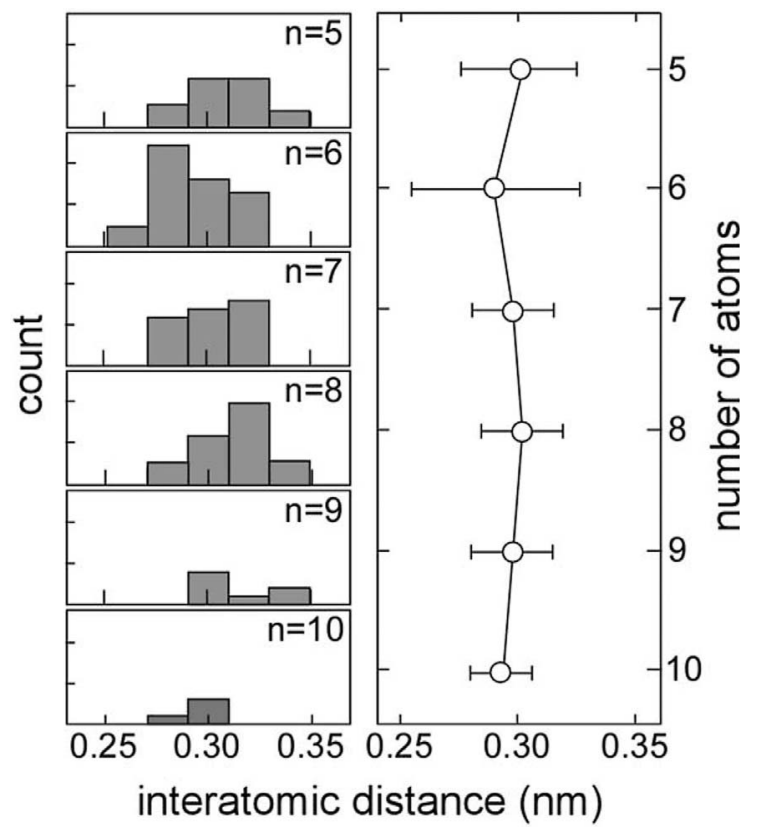

FIG. 8. Histograms of average interatomic distance of each ASW constructed of five to ten atoms (left side). The number of atoms in ASW for each histogram is indicated by $n$. The average interatomic distance estimated from each histogram is plotted on the right side as a function of the number of atoms.

states calculated by ab initio calculations. ${ }^{33,35,39,40,75} \mathrm{Al}-$ though light-element atoms are not observed in Figs. 9(b) and $9(\mathrm{c}),{ }^{76}$ the positions of Au atoms in the ASWs are also similar to the positions in ASWs with an incorporated oxygen atom and a hydrogen molecule, as revealed by ab initio calculations. $46,49,51$

Most of the ASWs in this study, regardless of interatomic distances, exhibit straight or slightly arched structures. As an exception, a few ASWs showed zigzag structures, which are predicted to be stable structures on the basis of $a b$ initio calculation. ${ }^{33,77-79}$ Such a zigzag ASW is shown in Fig. 10. The interatomic distances in this zigzag ASW are also similar to those of other ASWs. The bond angles near connecting points between the wire and electrodes are larger than those around the wire center. This result shows that the zigzag structure is not most stable at strained states under tensile condition, as pointed out by Garcia-Suarez et al. $^{78}$ and Ribeiro and Cohen. ${ }^{79}$

Figure 11 shows variations in the total length, the strain, and the force of a Au ASW during retraction as a function of time. At the times marked by arrowheads $\mathrm{A}-\mathrm{C}$ (hereafter, times $\mathrm{A}, \mathrm{B}$, and $\mathrm{C}$ ), the number of atoms increases to 8,9 , and 10, respectively. At the time indicated with arrowhead X, the ASW breaks. The strain increases gradually from time A to time $\mathrm{X}$. On the other hand, the force shows a sawtooth variation. The regions between two neighboring arrowheads represent the elastic elongation of the interatomic distance. Rapid decreases in force at the end of the regions imply the relaxation of the tensed structures. The relaxation occurs at a force of 1-2 nN. From the rates of increase in the length and force in each elastic region, the average force constant of the ASW is estimated to be $0.96-3.0 \mathrm{~N} / \mathrm{m}$. This force constant 

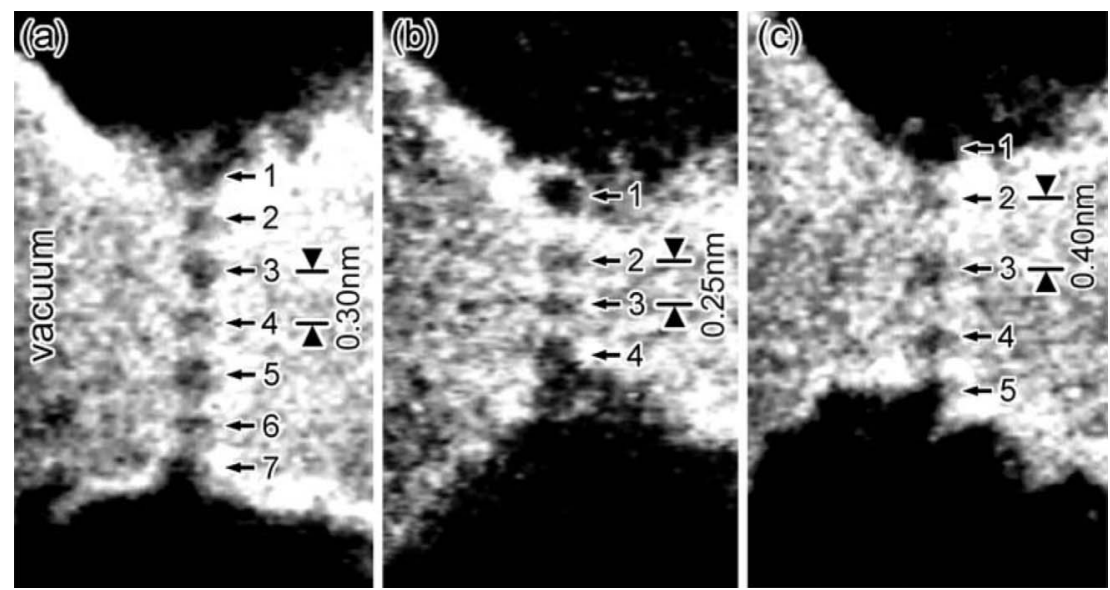

FIG. 9. High-resolution images of Au ASWs showing (a) average interatomic distance (0.30 nm), (b) minimum interatomic distance $(0.25 \mathrm{~nm})$, and (c) maximum interatomic distance $(0.40 \mathrm{~nm})$. The interatomic distances in (a) are $(1-2): 0.28 \mathrm{~nm} ;(2-3): 0.30 \mathrm{~nm} ;(3-4)$ : $0.30 \mathrm{~nm}$; (4-5): $0.30 \mathrm{~nm}$; (5-6): $0.30 \mathrm{~nm}$; (6-7): $0.26 \mathrm{~nm}$; (b) (1-2): $0.38 \mathrm{~nm}$; (2-3): $0.25 \mathrm{~nm}$; (3-4): $0.29 \mathrm{~nm}$; (c) $(1-2): 0.27 \mathrm{~nm}$; (2-3): $0.40 \mathrm{~nm}$; (3-4): $0.39 \mathrm{~nm}$; and (5-6): $0.28 \mathrm{~nm}$. The average interatomic distance of each ASW is (a) $0.29 \pm 0.02 \mathrm{~nm},($ b) $0.31 \pm 0.03 \mathrm{~nm}$, and (c) $0.34 \pm 0.03 \mathrm{~nm}$.

measured for the ASW constructed of eight to ten atoms corresponds to approximately one-fifth of the reported value measured previously by SPM for Au ASWs of undefined length $(5.8-15 \mathrm{~N} / \mathrm{m}){ }^{5}$ As shown in the HRTEM image in Fig. 1, it is clearly found that this stress relaxation, i.e., the recovery of the interatomic distance, is due to the introduction of a new atom from the plate to the wire. Similar sawtooth curves are observed for $\mathrm{Au}$ NCs during retraction, which are caused by the repetition of elastic deformation and yield due to slip. ${ }^{5,12,21,26}$ Although the shape of force-time curves of both the ASW and the NCs are similar, the yield mechanisms are different.

Since the length of the ASW increases during retraction, as shown in Fig. 11, to compare the force-elongation relation
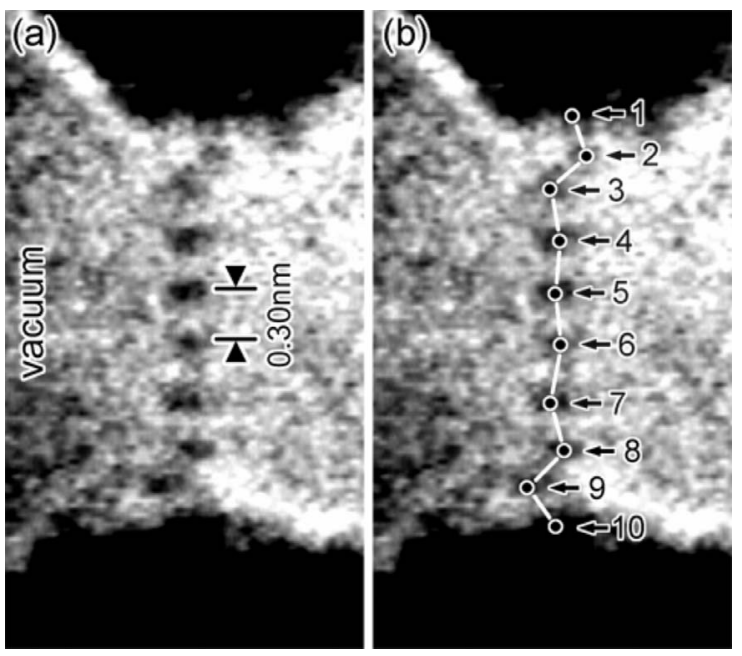

FIG. 10. (a) High-resolution image of zigzag ASW and (b) configuration of atoms. The interatomic distances in (a) are (1-2): $0.24 \mathrm{~nm}$; (2-3): $0.28 \mathrm{~nm}$; (3-4): $0.30 \mathrm{~nm}$; (4-5): $0.30 \mathrm{~nm}$; (5-6): $0.30 \mathrm{~nm}$; (6-7): $0.34 \mathrm{~nm}$; (7-8): $0.28 \mathrm{~nm}$; (8-9): $0.30 \mathrm{~nm}$; and (9-10): $0.28 \mathrm{~nm}$. The average interatomic distance of ASW is $0.29 \pm 0.01 \mathrm{~nm}$. of the ASW for each number of atoms, we plot the force as a function of strain, as shown in Fig. 12 along the right vertical axis. When we assume that the cross section of the ASW is defined by a constant, we can calculate the stress acting on the ASW. The stress is obtained by dividing the force by the cross-sectional area. Then, we derive the stress-strain rela-

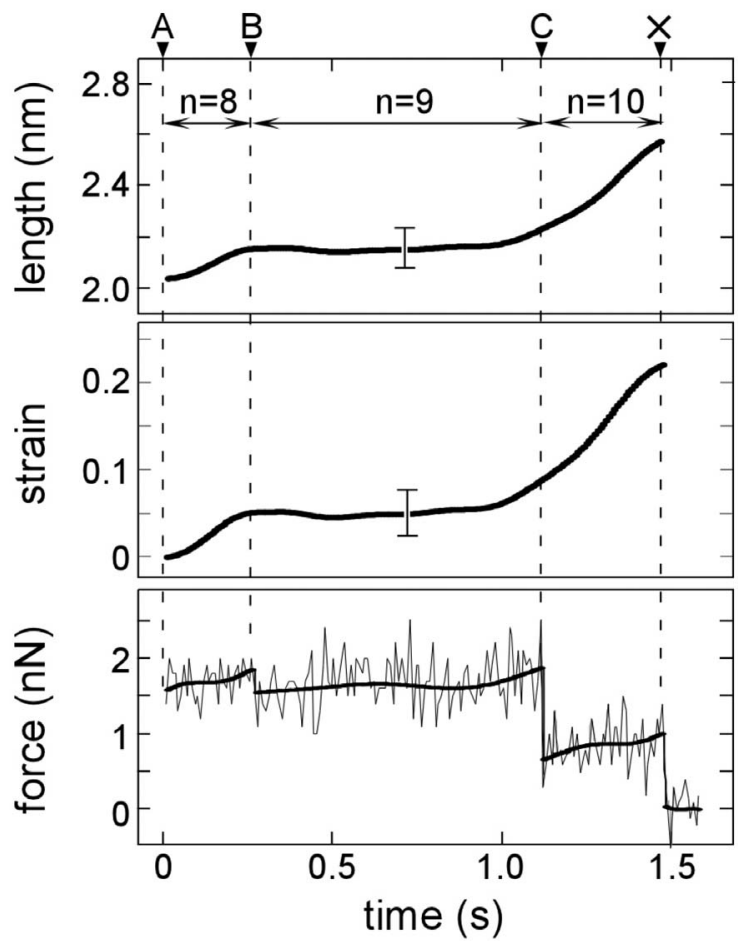

FIG. 11. Variations in total length, strain, and force of Au ASW during retraction as function of time. At times indicated by arrowheads $\mathrm{A}-\mathrm{C}$, the number of atoms $(n)$ increases to 8,9 , and 10 , respectively, and at the time indicated by arrowhead X, this ASW breaks. In the graph of force, the measured spectrum and its approximated curve are represented with the thin solid line and the bold solid line, respectively. 


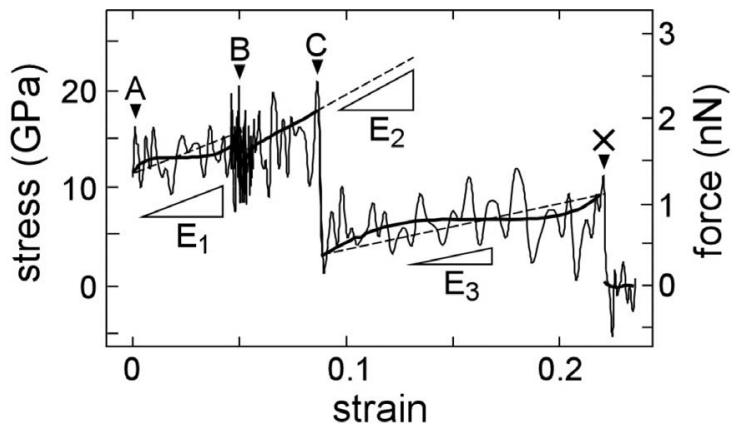

FIG. 12. Stress (force)-strain curve of Au ASW during retraction process observed in Fig. 11. The stress and force are shown on the left and right vertical axes, respectively. The measured spectrum and its approximated curve are represented by the thin solid line and the bold solid line, respectively. The strains indicated by A, B, and $\mathrm{C}$ correspond to those observed at times A, B, and C in Fig. 11, respectively. The broken lines represent the average inclinations, $E_{1}, E_{2}$, and $E_{3}$, in strain regions A-B, B-C, and C-X, respectively. $E_{1}, E_{2}$, and $E_{3}$ correspond to the average Young's modulus during the elongation of the ASW and are 86, 116, and $47 \mathrm{GPa}$, respectively. The interatomic distances at strains $\mathrm{A}, \mathrm{B}, \mathrm{C}$, and $\mathrm{X}$ are $0.34 \pm 0.01,0.31 \pm 0.01,0.29 \pm 0.01$, and $0.30 \pm 0.01 \mathrm{~nm}$, respectively.

tionship to analyze the mechanical properties of the ASWs on the basis of standard mechanics of materials. For such an estimation of the stress, we selected the cross-sectional area of one atom along the $\langle 110\rangle$ direction, i.e., $0.118 \mathrm{~nm}^{2}$, which was previously used for the estimation of the pressure acting on Au ASWs. ${ }^{5}$ In Fig. 12, we also show the stress on the left vertical axis. The stress at the relaxation due to the introduction of a new atom is $8-17 \mathrm{GPa}$. This value is recognized as the strength of the ASW and is 58-120 times that of coarsegrained $\mathrm{Au}$ crystals and two to six times that of $\mathrm{Au} \mathrm{NCs}$ $(2-8 \mathrm{GPa})^{5,12,21,58}$ The continuous elongation occurs between the two neighboring stress-relaxation events. The amount of strain between the two difference events is 0.040.13. During the one continuous elastic elongation process, we can estimate its stress-strain rate that corresponds to Young's modulus. The average Young's modulus of the ASW in the strain region between times A-B, B-C, and C-X in Fig. 12 is $E_{1}=86, E_{2}=116$, and $E_{3}=47 \mathrm{GPa}$, respectively. These values are similar to Young's moduli of coarse-grained $\mathrm{Au}$ single crystals along the $\langle 110\rangle,\langle 111\rangle$, and $\langle 100\rangle$ directions. ${ }^{80}$

As shown in Fig. 12, Young's modulus fluctuates by $35 \%-60 \%$. Young's modulus is estimated as a constant of proportionality between stress and strain in each elastic elongation process. The elongation behavior is dominated by the atomic configration of an ASW and the connecting tips at the start of each elongation process, i.e., at the extraction of an atom from the tip. In particular, the variation in the atomic configration of the tips at each extraction of an atom is larger than that of the ASW, as seen in Fig. 1. Thus, Young's modulus of each elongation process does not necessarily decrease as the length increases. In this study, features of mechanical parameters appearing with increasing the wire length, e.g., a monotone decrease in Young's modulus or even-odd oscillation in force, are not observed. To investigate such features, it is required to increase atomic diffusion to recover tip shape.

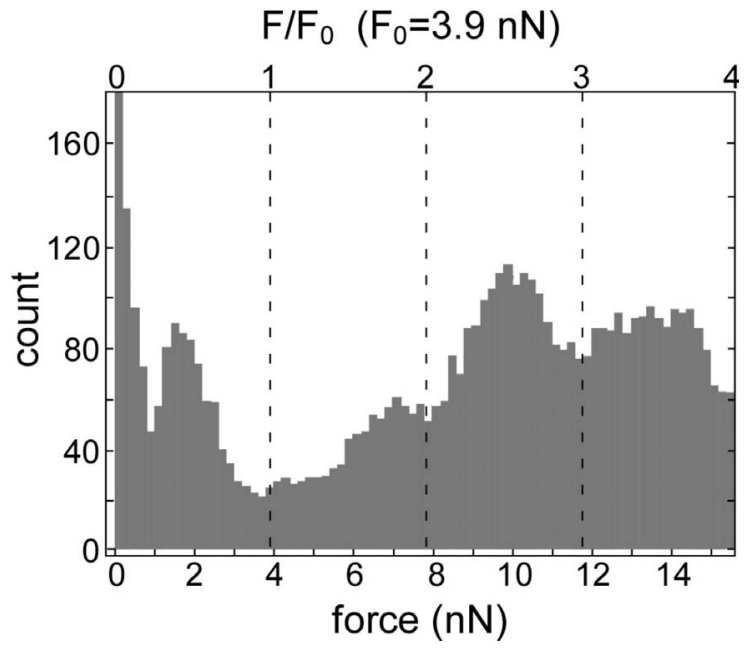

FIG. 13. Histogram of force values measured during retraction process of $40 \mathrm{Au}$ NCs biased by $13 \mathrm{mV}$ between tip and plate. The force is expressed in units of $F_{0}(=3.9 \mathrm{nN})$ (upper lateral axis).

The stress acting on the observed ASWs and the interatomic distance were measured for each tensed structure. In Fig. 12, the maximum stress is observed at the end of each elastic elongation region between times A-B, B-C, and C-X and is $16 \mathrm{GPa}$ at strain at time $\mathrm{B}, 18 \mathrm{GPa}$ at time $\mathrm{C}$, and $9.4 \mathrm{GPa}$ at time $\mathrm{X}$. The average interatomic distances at these strain states are $0.31 \pm 0.01,0.29 \pm 0.01$, and $0.30 \pm 0.01 \mathrm{~nm}$ at strain at times $\mathrm{B}, \mathrm{C}$, and $\mathrm{X}$, respectively. The amount of strain required to reduce the ASW to a stressfree state is calculated, assuming that a simple linear relationship holds between the stress $(\sigma)$ and the strain $(\varepsilon)$ with Young's modulus $(E)$ as a coefficient, i.e., $\sigma=E \varepsilon$. Using the average Young's moduli $E_{1}, E_{2}$, and $E_{3}$, the amount of strain needed to recover from each tensed state is calculated to be $0.18,0.15$, and 0.20 , which corresponds to the elastic limit of the ASW. From these strain values and the interatomic distances, the stress-free interatomic distances are calculated to be $0.26 \pm 0.02,0.26 \pm 0.01$, and $0.25 \pm 0.01 \mathrm{~nm}$ for each strain region, assuming that each atomic bond in the ASW elongates with the same Young's modulus. These interatomic distances are similar to that measured by MCBJTs and obtained by $a b$ initio calculation. ${ }^{14,32,34,81-83}$

Figure 13 shows a histogram of force values measured during retraction processes of $\mathrm{Au}$ NCs. For the construction of this histogram, 40 force-time curves of Au NCs were selected, during which the formation of ASWs was confirmed by HRTEM. In Fig. 13, peaks are observed at 1.6, 7.1, 10.0, and $13.3 \mathrm{nN}$. The HRTEM observation showed that the first peak at $1.6 \mathrm{nN}$ with a full width at half maximum of $0.7 \mathrm{nN}$ is dominated by ASW formation, as shown in Fig. 12. As shown in Fig. 1(1), the ASW disappears owing to the migration of the constituent atoms to the tip and the electrode after fracture. Thus, a tensile force of $1.6 \pm 0.7 \mathrm{nN}$ is needed to observe the ASWs. The increase in stability of straight ASWs at strained states is also pointed out previously on the basis of $a b$ initio calculation. ${ }^{79}$ In this study, we changed the direction of force from retraction to compression. Most of the longer ASWs, as observed in Fig. 1, broke at the change of direction. This instability is explained owing to the reduction 
in tensile force to around zero. On the other hand, for shorter ASWs with a few atoms in length, they kept straight shapes during cycles of retraction and compression, showing that the constituent atoms of ASWs diffused back to the electrodes. This suggests that larger tensile forces are needed to maintain longer ASWs.

The other higher peaks were caused by NCs with a width of two to four atoms. In contrast to the conductance histogram seen in Fig. 7, the peak positions in the force histogram are not quantized. However, the local minimum positions are found at integer multiples of a force unit $F_{0}$, as indicated by the broken lines in Fig. 13. $F_{0}$ is estimated to be $3.9 \mathrm{nN}$. This cycle regularity of the local minimum position was not observed over $4 F_{0}$.

\section{DISCUSSION}

\section{A. Structure and stability}

As shown in Fig. 4, the atomic configurations of the observed ASW are classified into two types: the slightly arched configuration seen in Figs. 4(e)-4(g) and almost straight configuration seen in Figs. 4(h)-4(k). The arched configuration is observed even during retraction. When the arched configuration was present, the retraction speed was recorded as $0.077 \mathrm{~nm} / \mathrm{s}$. During the observation of the straight atom configuration, the recorded speed increased by $250 \%$. The ASW grows owing to the migration of atoms from the tip and the plate to the wire part. Thus, the ASW becomes arched when the retraction speed is slower than the migration speed, and the ASW approaches a straight configuration as these two speeds become similar. Taking into account the observed average interatomic distance of $0.30 \mathrm{~nm}$, the average speed of atoms migrating into the ASW is estimated to be $0.26-0.63$ atoms / s.

Tsutsui et al. has shown that the Au contacts of $\sim 0.3 \mathrm{~nm}$ in length corresponding to $1 G_{0}$ can survive $10 \mathrm{~s}$ or longer at room temperature when the contact retraction speed is reduced to be as low as $0.8 \mathrm{pm} / \mathrm{s}^{84}$ They argue that the retraction speed comparable with the surface diffusion rate of $\mathrm{Au}$ might help the formation of long-lived $1 G_{0}$ contacts. Their results thus support the our estimation of the atom migration rate from the straightening of curved ASWs at higher retraction speed.

The interatomic distance of Au ASWs, measured in a cryogenic vacuum at $4.2 \mathrm{~K}$ by MCBJTs, was reported to be $0.25 \pm 0.02 \mathrm{~nm} . .^{14,82,85}$ This value is in good agreement with the calculated interatomic distances of structurally stabilized Au ASWs. ${ }^{32,34,81,83,86,87}$ On the other hand, HRTEM observations by Ohnishi et al. ${ }^{41}$ and Rodrigues and Ugarte ${ }^{43}$ show longer distances of $0.33-0.40 \mathrm{~nm}$ for Au ASWs produced by intense electron beam processing, during which the force acting was not estimated. In this study, the interatomic distance is measured to be $0.25-0.40 \mathrm{~nm}$ with an average interatomic distance of $0.30 \pm 0.02 \mathrm{~nm}$. This average interatomic distance is expanded by a strain of $0.15-0.20$ under a tensile force of $1.6 \pm 0.7 \mathrm{nN}$ when we assume that each atomic bond of the ASW expands homogenously, as in the state seen in Fig. 9(a). Thus, the average interatomic distance in the stress-free state is estimated to be reduced to $0.26 \pm 0.02 \mathrm{~nm}$.
On the other hand, the ASWs show inhomogeneous interatomic distances, as observed in Figs. 9(b) and 9(c) and Fig. 10. This configuration is comparable to both the $\mathrm{Au}$ atom positions of pure $\mathrm{Au}$ dimer-coupled states $33,35,39,40,75$ and $\mathrm{Au}-$ impurity complex states. ${ }^{34,35,39,46,49,51}$ Light-element atoms such as hydrogen, carbon, and oxygen, which are constituent atoms of major residual gaseous molecules in the specimen chamber, are not imaged by HRTEM. ${ }^{76}$ Thus, these two states cannot be distinguished from the analysis of the $\mathrm{Au}$ atom positions. It is noted that the duration of pure $\mathrm{Au}$ ASWs, which are measured by SPM, is, at most, $\sim 2 \mathrm{~ms}$ at a retraction speed of $\sim 10 \mu \mathrm{m} / \mathrm{s} .{ }^{69,72,73,88}$ According to $a b$ initio calculations, pure Au ASWs with longer interatomic distances occurring at dimerization are unstable because the distances are similar to or exceed the fracture length. ${ }^{33,40,77,83} \mathrm{In}$ contrast, Au ASWs are strengthened owing to the incorporation and absorption of hydrogen, boron, carbon, oxygen, and sulfur atoms. ${ }^{46,50,51}$ The present ASWs show a duration time beyond $10 \mathrm{~s}$. In addition, the longest ASWs in this study are twice as long as the fracture length predicted by Novaes et $a l$. on the basis of $a b$ initio calculations. ${ }^{51}$ Thus, from this increase in duration, it is concluded that the stable ASWs observed in this study are complexed with light-element atoms.

\section{B. Conductance}

As shown in Figs. 5 and 6, the ASW shows a conductance peak of approximately $1 G_{0}$ when the number of constituent atoms is 5 , and the conductance decreases to less than $0.1 G_{0}$ as the atom number increases. The length of the ASW constructed by five to ten atoms is $1.2-2.7 \mathrm{~nm}$, as shown in Fig. 5. This length is shorter than a mean free path of 3-6 nm estimated for Au ASWs at $4.2 \mathrm{~K}$, although we should take into account the difference between the temperatures at the time of measurement. ${ }^{74,89}$ We consider structural variation in the ASW to interpret the decrease in conductance from $1 G_{0}$ to less than $0.1 G_{0}$. It has been reported that the expansion of $\mathrm{Au}$ ASWs causes dimerization; an expansion of the interatomic distance to $0.32 \mathrm{~nm}$ contributes to the decrease from $1 G_{0}$ to less than $0.1 G_{0} \cdot{ }^{33}$ However, the interatomic distance at this expansion is close to the distance at fracture; the ASWs are unstable. ${ }^{33,40,75}$ In the conductance-separation distance relations measured previously for Au NCs in ultrahigh vacuum, a plateau at $1 G_{0}$ with a slight negative coefficient is observed; however, the conductance decreases by, at most, $\sim 0.1 G_{0} .{ }^{5}$ These previous results concerning the stability and conductance of the expanded pure Au ASWs contradicts those of the ASWs, showing $0.1 G_{0}$ in this study; the $0.1 G_{0}$ state is maintained for at least $5 \mathrm{~s}$, as seen in times (f)-(k) in Fig. 5. It is also noted in previous ab initio calculations that the conductance decreases gradually during the continuous elongation of the interatomic distance; no peak is formed in the conductance histogram in the range below $1 G_{0} \cdot{ }^{33} \mathrm{How}-$ ever, in this study, the $0.5 G_{0}$ peak is observed, as shown in Fig. 7. The decrease in conductance also results from the combination of Au ASWs with impurity atoms and gaseous molecules. ${ }^{34,35,39,47,49-51,82,90}$ In particular, the conductance decreases to less than $0.1 G_{0}$ upon the incorporation of an 
oxygen atom in, or the absorption of a hydrogen molecule on, a Au ASW, as indicated by ab initio calculations. ${ }^{34,49} \mathrm{It}$ is also reported that a $0.5 G_{0}$ peak is observed in conductance measurements of Au NCs in a hydrogen gas by MCBJT. ${ }^{47}$ The stability of ASWs increases upon the incorporation of impurity atoms and molecules. ${ }^{46,50,51}$ Therefore, as per the atomic configuration and stability of the present ASWs, the decrease in conductance is likewise explained by the combination of the impurity atoms and molecules in ASWs.

\section{Mechanical properties}

As shown in Fig. 11, the force-time relationship of the ASW shows a sawtooth variation corresponding to cycles of a steadily increasing force region in the elastic elongation process and a rapid force decrease due to stress relaxation by the extraction of a new atom from the tip and the plate. In Fig. 13, the peak at $1.6 \mathrm{nN}$ is related to the count accumulation for the ASWs. For tensile deformation of thicker contacts, i.e., NCs, a sawtooth variation is also observed as cycles of elastic elongation and its stress relaxation. ${ }^{21,58}$ Slip and liquidlike atomic migration are involved in the stress relaxation. ${ }^{15,16,18,36,54}$ The count accumulation from elastic elongation for a similar atomic configuration is represented as a peak in the force histogram. In Fig. 13, the peaks at 7.1, 10.0 , and $13.3 \mathrm{nN}$ result from the count accumulation for NCs of cross-sectional areas of two to four atoms. The force level of an elastic elongation process is determined by the structure and elongation properties of the ASW, the connecting tip, and the plate. The atomic configuration of the total structure, the orientation, and the convergent angle of the tip and plate differ among NCs. As a result, the force values in elastic elongation processes for NCs with undefined orientational relationships are dispersed in the force histogram. Thus, the peaks in the force histogram are not necessarily formed with the same interval of force, as shown in Fig. 13. On the other hand, the force values at local-minimum positions in the histogram are found at integer multiples of $F_{0}$. The counts in the force histogram increase for a force range corresponding to the elastic elongation process, whereas the counts decrease at a gap between two force ranges of the elastic elongation processes with neighboring levels. Therefore, the local minimum in the gap represents a statistical maximum force appearing upon the stress relaxation of NCs with a minimum cross section. The stress relaxation occurs at the weakest region of a $\mathrm{NC}$, which is the stress-concentrated thinnest region of each structure, i.e., the region of minimum cross-sectional area. Until fracture, the stress-strain relationship of a NC is determined by the mechanical properties of its total structure. However, the force at the stress relaxation is dominated by the strength of the few atomic bonds in this thinnest region. Therefore, it is deduced that the force at the local minimum positions at integer multiples in the histogram represents the statistical strength of the atomic bonds of integer atoms at the minimum cross section.

The force at fracture of Au ASWs is measured, in a cryogenic vacuum, by SPM to be $1.5 \pm 0.2 \mathrm{nN}, 5,12$ and is estimated theoretically for impurity-free states to be $0.3-2.1 \mathrm{nN} .{ }^{30,51,81,83,91-94} \mathrm{In}$ this study, the force at the first peak in the histogram in Fig. 13, $1.6 \pm 0.7 \mathrm{nN}$, is comparable to such previously measured and estimated values at fracture. However, the force at the first peak corresponds to the force during the elastic elongation rather than the force at fracture. As discussed above, we deduced that the force at the first local minimum in the force histogram, i.e., $1 F_{0}=3.9 \mathrm{nN}$, represents the statistical strength of the ASW. This value is higher than the fracture forces reported previously for pure $\mathrm{Au}$ ASWs. The stability of ASWs increases upon the incorporation of impurity atoms and molecules. ${ }^{46,50,51}$ In particular, owing to the incorporation of an oxygen atom in a $\mathrm{Au}$ ASW, the calculated force at fracture increases from $1.7 \mathrm{nN}$ for a pure state to more than $2.3 \mathrm{nN} .{ }^{51}$ It is expected that the force at fracture increases as the number of oxygen atoms increases. The present ASWs are observed for $\sim 10 \mathrm{~s}$. The probability of incorporation increases as time passes. The incorporation of a few oxygen atoms is inferred to be a possible factor in the higher force observed in this study.

The stress-strain curves for ASWs help to supply information concerning the relationship between the interatomic spacing and the mechanical parameters when the shape of ASWs is straight and the interatomic spacing is almost homogeneous. The expansion of interatomic spacing is expressed by strain, and force, or strength is shown as a function of strain for each state in an ASW.

In this discussion, we focus on the effects of oxygen atoms and hydrogen molecules. This is because, in the vacuum in the specimen chamber of our microscope, hydrogen and oxygen molecules are major residual gaseous molecules, which are easily absorbed on and incorporated with $\mathrm{Au}$ ASWs. It is also desirable to consider the effects of other residual gaseous molecules and impurity atoms. For example, carbon atoms exist as carbon monoxide, carbon dioxide, and hydrocarbon molecules in the vacuum. As pointed out by Legoas et al., the interatomic distance of Au ASWs observed by HRTEM is explained by incorporation with caron atoms. ${ }^{45,95,96}$ In this study, we could not observe single carbon atoms on Au ASWs. However, in contrast with hydrogen and oxygen, if carbon atoms are included in ASWs, they are solidified as carbon clusters, e.g., fullerenes or graphenes, on the surfaces of ASWs and the tips. ${ }^{62,66} \mathrm{We}$ can discuss the possibility of carbon impurity in the ASWs by reference to theoretical studies on the structure, mechanical, and electrical properties if we observe such cluster formation.

\section{CONCLUSION}

We directly observed, by in situ HRTEM, the formation of $\mathrm{Au}$ ASWs and their growth process up to a ten-atom length. It was found that the duration of the long ASWs exceeds $10 \mathrm{~s}$. The conductance and force of the stable long ASWs were examined through simultaneous measurements with HRTEM. It was concluded from the simultaneous observation that the observed stable ASWs were complexed with light-element atoms. 
The average interatomic distance of the ASWs was measured to be $0.30 \pm 0.5 \mathrm{~nm}$ during loading at a tensile force of $1.6 \pm 0.7 \mathrm{nN}$. On the basis of the measured stress-strain relationships, the strength and Young's modulus of the ASWs were derived. From these structural and mechanical values, the strain for the observed ASWs was estimated to be $20 \%$. The ASWs constructed with five atoms showed a stable $1.0 G_{0}$ state during the conductance fluctuation between 0.1 and $1.5 G_{0}$, whereas the conductance decreased to less than $0.1 G_{0}$ as the number of atoms exceeded 5 .

As shown in this study, the results of in situ HRTEM provide experimental evidence on atomically localized structures of the ASWs and clearly indicate a direct link of the structures to mechanical and electrical properties. By selecting the spectra of conductance and force on the basis of the confirmation of the formation of ASWs, we can refine the histograms of the spectra and elucidate the details of the peculiar nature of ASWs.

\section{ACKNOWLEDGMENTS}

The author would like to thank Takao Sumi and Katsuyoshi Kumazawa of Nagoya University for help with the production of the electron microscope demonstrated in this study, Satoru Fujisawa, Shinya Sasaki, Akira Yabe, and Yuji Enomoto of the National Institute of Advanced Industrial Science and Technology, and Shunji Deguchi and Mikio Naruse of JEOL for cooperation in developing the nanonewton force measurement system. The author thanks Kanji Yamada, Kazue Hosoki, Shinjiro Umehara, and Hajime Ohmi of Nagoya University for help with the image analysis. This work was partly supported by funds for the Special Research Project on Nanoscience and the University Research Projects of the University of Tsukuba, and Grants-in-Aid from the Ministry of Education, Culture, Sports, Science and Technology, Japan (Grants Nos. 16656040 and 18310075). *kizuka@ims.tsukuba.ac.jp

${ }^{1}$ C. J. Muller, J. M. van Ruitenbeek, and L. J. de Jongh, Phys. Rev. Lett. 69, 140 (1992).

${ }^{2}$ J. I. Pascual, J. Méndez, J. Gómez-Herrero, A. M. Baró, N. García, and V. T. Binh, Phys. Rev. Lett. 71, 1852 (1993).

${ }^{3}$ N. Agraït, J. G. Rodrigo, and S. Vieira, Phys. Rev. B 47, 12345 (1993).

${ }^{4}$ J. M. Krans, C. J. Muller, I. K. Yanson, T. C. M. Govaert, R. Hesper, and J. M. van Ruitenbeek, Phys. Rev. B 48, 14721 (1993).

${ }^{5}$ G. Rubio, N. Agraït, and S. Vieira, Phys. Rev. Lett. 76, 2302 (1996)

${ }^{6}$ C. Sirvent, J. G. Rodrigo, S. Vieira, L. Jurczyszyn, N. Mingo, and F. Flores, Phys. Rev. B 53, 16086 (1996).

${ }^{7}$ J. L. Costa-Krämer, Phys. Rev. B 55, R4875 (1997).

${ }^{8}$ J. L. Costa-Krämer, N. García, P. García-Mochales, P. A. Serena, M. I. Marqués, and A. Correia, Phys. Rev. B 55, 5416 (1997).

${ }_{9}^{9}$ A. I. Yanson, G. R. Bollinger, H. E. van den Brom, N. Agraït, and J. M. van Ruitenbeek, Nature (London) 395, 783 (1998).

${ }^{10}$ E. Scheer, N. Agraï, J. C. Cuevas, A. L. Yeyati, B. Ludoph, A. Martín-Rodero, G. R. Bollinger, J. M. Ruitenbeek, and C. Urbina, Nature (London) 394, 154 (1998).

${ }^{11}$ B. Ludoph, N. van der Post, E. N. Bratus, E. V. Bezuglyi, V. S. Shumeiko, G. Wendin, and J. M. van Ruitenbeek, Phys. Rev. B 61, 8561 (2000).

${ }^{12}$ G. Rubio-Bollinger, S. R. Bahn, N. Agraït, K. W. Jacobsen, and S. Vieira, Phys. Rev. Lett. 87, 026101 (2001).

${ }^{13}$ S. K. Nielsen, M. Brandbyge, K. Hansen, K. Stokbro, J. M. van Ruitenbeek, and F. Besenbacher, Phys. Rev. Lett. 89, 066804 (2002).

${ }^{14}$ C. Untiedt, A. I. Yanson, R. Grande, G. Rubio-Bollinger, N. Agraït, S. Vieira, and J. M. van Ruitenbeek, Phys. Rev. B 66, 085418 (2002).

${ }^{15}$ U. Landman, W. D. Luedtke, N. A. Burnham, and R. Colton, Science 248, 454 (1990).

${ }^{16}$ M. Brandbyge, J. Schiøtz, M. R. Sørensen, P. Stoltze, K. W. Jacobsen, J. K. Nørskov, L. Olesen, E. Laegsgaard, I. Stens- gaard, and F. Besenbacher, Phys. Rev. B 52, 8499 (1995).

${ }^{17}$ L. Olesen, E. Lægsgaard, I. Stensgaard, F. Besenbacher, J. Schiøtz, P. Stoltze, K. W. Jacobsen, and J. K. Nørskov, Phys. Rev. Lett. 72, 2251 (1994).

${ }^{18}$ A. M. Bratkovsky, A. P. Sutton, and T. N. Todorov, Phys. Rev. B 52, 5036 (1995).

${ }^{19}$ J. L. Costa-Krämer, N. García, P. García-Mochales, and P. A. Serena, Surf. Sci. 342, L1144 (1995).

${ }^{20}$ J. M. Krans, J. M. van Ruitenbeek, V. V. Fisun, I. K. Yanson, and L. J. de Jongh, Nature (London) 375, 767 (1995).

${ }^{21}$ N. Agraït, G. Rubio, and S. Vieira, Phys. Rev. Lett. 74, 3995 (1995).

22 J. I. Pascual, J. Méndez, J. Gómez-Herrero, A. M. Baró, N. Garcia, U. Landman, W. D. Luedtke, E. N. Bogachek, and H.-P. Cheng, Science 267, 1793 (1995).

${ }^{23}$ C. Zhou, C. J. Muller, M. R. Deshpande, J. W. Sleight, and M. A. Reed, Appl. Phys. Lett. 67, 1160 (1995).

${ }^{24}$ U. Landman, W. D. Luedtke, B. E. Salisbury, and R. L. Whetten, Phys. Rev. Lett. 77, 1362 (1996).

${ }^{25}$ A. G. Scherbakov, E. N. Bogachek, and U. Landman, Phys. Rev. B 53, 4054 (1996).

${ }^{26}$ A. Stalder and U. Dürig, J. Vac. Sci. Technol. B 14, 1259 (1996).

${ }^{27}$ C. Untiedt, G. Rubio, S. Vieira, and N. Agraït, Phys. Rev. B 56, 2154 (1997).

${ }^{28}$ A. Correia and N. García, Phys. Rev. B 55, 6689 (1997).

${ }^{29}$ H. Yasuda and A. Sakai, Phys. Rev. B 56, 1069 (1997).

${ }^{30}$ M. R. Sørensen, M. Brandbyge, and K. W. Jacobsen, Phys. Rev. B 57, 3283 (1998).

${ }^{31}$ M. Brandbyge, N. Kobayashi, and M. Tsukada, Phys. Rev. B 60, 17064 (1999).

${ }^{32}$ E. G. Emberly and G. Kirczenow, Phys. Rev. B 60, 6028 (1999).

${ }^{33}$ M. Okamoto and K. Takayanagi, Phys. Rev. B 60, 7808 (1999).

${ }^{34}$ S. R. Bahn, N. Lopez, J. K. Nørskov, and K. W. Jacobsen, Phys. Rev. B 66, 081405(R) (2002).

${ }^{35}$ H. Mehrez, A. Wlasenko, B. Larade, J. Taylor, P. Grütter, and H. Guo, Phys. Rev. B 65, 195419 (2002). 
${ }^{36}$ T. N. Todorov and A. P. Sutton, Phys. Rev. Lett. 70, 2138 (1993).

${ }^{37}$ J. A. Torres, J. I. Pascual, and J. J. Sáenz, Phys. Rev. B 49, 16581 (1994)

${ }^{38}$ M. Dreher, F. Pauly, J. Heurich, J. C. Cuevas, E. Scheer, and P. Nielaba, Phys. Rev. B 72, 075435 (2005).

${ }^{39}$ H. Häkkinen, R. N. Barnett, and U. Landman, J. Phys. Chem. B 103, 8814 (1999).

${ }^{40}$ J. A. Torres, E. Tosatti, A. Dal Corso, F. Ercolessi, J. J. Kohanoff, F. D. Di Tolla, and J. M. Soler, Surf. Sci. 426, L441 (1999).

${ }^{41}$ H. Ohnishi, Y. Kondo, and K. Takayanagi, Nature (London) 395, 780 (1998).

${ }^{42}$ T. Kizuka, S. Umehara, and S. Fujisawa, Jpn. J. Appl. Phys., Part 2 40, L71 (2001).

${ }^{43}$ V. Rodrigues and D. Ugarte, Phys. Rev. B 63, 073405 (2001).

${ }^{44}$ Y. Takai, T. Kawasaki, Y. Kimura, T. Ikuta, and R. Shimizu, Phys. Rev. Lett. 87, 106105 (2001).

${ }^{45}$ S. B. Legoas, D. S. Galvão, V. Rodrigues, and D. Ugarte, Phys. Rev. Lett. 88, 076105 (2002).

${ }^{46}$ F. D. Novaes, A. J. R. da Silva, E. Z. da Silva, and A. Fazzio, Phys. Rev. Lett. 90, 036101 (2003).

${ }^{47}$ S. Csonka, A. Halbritter, G. Mihály, E. Jurdik, O. I. Shklyarevskii, S. Speller, and H. van Kempen, Phys. Rev. Lett. 90, 116803 (2003)

${ }^{48}$ S. Csonka, A. Halbritter, G. Mihály, O. I. Shklyarevskii, S. Speller, and H. van Kempen, Phys. Rev. Lett. 93, 016802 (2004).

${ }^{49}$ R. N. Barnett, H. Häkkinen, A. G. Scherbakov, and U. Landman, Nano Lett. 4, 1845 (2004).

${ }^{50}$ S. Csonka, A. Halbritter, and G. Mihaly, Phys. Rev. B 73, 075405 (2006).

${ }^{51}$ F. D. Novaes, A. J. R. da Silva, E. Z. da Silva, and A. Fazzio, Phys. Rev. Lett. 96, 016104 (2006).

${ }^{52}$ T. Kizuka and N. Tanaka, Philos. Mag. Lett. 69, 135 (1994).

${ }^{53}$ T. Kizuka, K. Yamada, S. Deguchi, M. Naruse, and N. Tanaka, Phys. Rev. B 55, R7398 (1997).

${ }^{54}$ T. Kizuka, Phys. Rev. Lett. 81, 4448 (1998).

${ }^{55}$ T. Kizuka, Phys. Rev. B 57, 11158 (1998).

${ }^{56}$ D. Erts, H. Olin, L. Ryen, E. Olsson, and A. Thölén, Phys. Rev. B 61, 12725 (2000).

${ }^{57}$ V. Rodrigues, T. Fuhrer, and D. Ugarte, Phys. Rev. Lett. 85, 4124 (2000).

${ }^{58}$ T. Kizuka, H. Ohmi, T. Sumi, K. Kumazawa, S. Deguchi, M. Naruse, S. Fujisawa, S. Sasaki, A. Yabe, and Y. Enomoto, Jpn. J. Appl. Phys., Part 2 40, L170 (2001).

${ }^{59}$ D. Erts, A. Lôhmus, R. Lômus, and H. Olin, Appl. Phys. A: Mater. Sci. Process. 72, S71 (2001).

${ }^{60}$ D. Erts, A. Lôhmus, R. Lômus, H. Olin, A. V. Pokropivny, L. Ryen, and K. Svensson, Appl. Surf. Sci. 188, 460 (2002).

${ }^{61}$ T. Kizuka, Y. Takatani, K. Asaka, and R. Yoshizaki, Phys. Rev. B 72, 035333 (2005).

${ }^{62}$ K. Asaka and T. Kizuka, Phys. Rev. B 72, 115431 (2005).

${ }^{63}$ T. Matsuda and T. Kizuka, Jpn. J. Appl. Phys., Part 2 45, L1337 (2006).

${ }^{64}$ M. Ryu and T. Kizuka, Jpn. J. Appl. Phys., Part 1 45, 8952 (2006).

${ }^{65}$ T. Kizuka and Y. Takatani, Jpn. J. Appl. Phys., Part 1 46, 5706
(2007).

${ }^{66}$ K. Asaka, R. Kato, R. Yoshizaki, K. Miyazawa, and T. Kizuka, Phys. Rev. B 76, 113404 (2007).

${ }^{67}$ T. Matsuda and T. Kizuka, Jpn. J. Appl. Phys., Part 1 46, 4370 (2007).

${ }^{68}$ K. Ishizuka, Ultramicroscopy 5, 55 (1980).

${ }^{69}$ A. Fujii, M. Tsutsui, S. Kurokawa, and A. Sakai, Phys. Rev. B 72, 045407 (2005).

${ }^{70}$ R. H. M. Smit, C. Untiedt, and J. M. van Ruitenbeek, Nanotechnology 15, S472 (2004).

${ }^{71}$ G. A. Sallivan, J. Phys. Chem. Solids 28, 347 (1967).

${ }^{72}$ K. Hansen, S. K. Nielsen, M. Brandbyge, E. Lægsgaard, I. Stensgaard, and F. Besenbacher, Appl. Phys. Lett. 77, 708 (2000).

${ }^{73}$ K. Hansen, S. K. Nielsen, E. Lægsgaard, I. Stensgaard, and F. Besenbacher, Rev. Sci. Instrum. 71, 1793 (2000).

${ }^{74}$ B. Ludoph and J. M. van Ruitenbeek, Phys. Rev. B 61, 2273 (2000).

${ }^{75}$ L. De Maria and M. Springborg, Chem. Phys. Lett. 323, 293 (2000).

${ }^{76}$ K. Koizumi, Y. Oshima, Y. Kondo, and K. Takayanagi, Ultramicroscopy 88, 17 (2001).

${ }^{77}$ D. Sánchez-Portal, E. Artacho, J. Junquera, P. Ordejón, A. García, and J. M. Soler, Phys. Rev. Lett. 83, 3884 (1999).

${ }^{78}$ V. M. García-Suárez, A. R. Rocha, S. W. Bailey, C. J. Lambert, S. Sanvito, and J. Ferrer, Phys. Rev. Lett. 95, 256804 (2005).

${ }^{79}$ F. J. Ribeiro and M. L. Cohen, Phys. Rev. B 68, 035423 (2003).

${ }^{80}$ T. H. Courtney, Mechanical Behavior of Materials (Waveland, Long Grove, 2000).

${ }^{81}$ S. R. Bahn and K. W. Jacobsen, Phys. Rev. Lett. 87, 266101 (2001).

${ }^{82}$ W. H. A. Thijssen, D. Marjenburgh, R. H. Bremmer, and J. M. van Ruitenbeek, Phys. Rev. Lett. 96, 026806 (2006).

${ }^{83}$ A. Ayuela, M. J. Puska, R. M. Nieminen, and J. A. Alonso, Phys. Rev. B 72, 161403(R) (2005).

${ }^{84}$ M. Tsutsui, K. Shoji, M. Taniguchi, and T. Kawai, Nano Lett. 8, 345 (2008).

${ }^{85}$ R. H. M. Smit, C. Untiedt, G. Rubio-Bollinger, R. C. Segers, and J. M. van Ruitenbeek, Phys. Rev. Lett. 91, 076805 (2003).

${ }^{86}$ H. Häkkinen, R. N. Barnett, A. G. Scherbakov, and U. Landman, J. Phys. Chem. B 104, 9063 (2000).

${ }^{87}$ A. Delin and E. Tosatti, Phys. Rev. B 68, 144434 (2003).

${ }^{88}$ R. Suzuki, M. Tsutsui, D. Miura, S. Kurokawa, and A. Sakai, Jpn. J. Appl. Phys., Part 1 46, 3694 (2007).

${ }^{89}$ B. Ludoph, M. H. Devoret, D. Esteve, C. Urbina, and J. M. van Ruitenbeek, Phys. Rev. Lett. 82, 1530 (1999).

${ }^{90}$ C. Z. Li, H. Sha, and N. J. Tao, Phys. Rev. B 58, 6775 (1998).

${ }^{91}$ J. A. Torres and J. J. Sáenz, Phys. Rev. Lett. 77, 2245 (1996).

${ }^{92}$ C. A. Stafford, D. Baeriswyl, and J. Burki, Phys. Rev. Lett. 79, 2863 (1997).

${ }^{93}$ E. Z. da Silva, A. J. R. da Silva, and A. Fazzio, Phys. Rev. Lett. 87, 256102 (2001).

${ }^{94}$ S. P. Javis, M. A. Lantz, H. Ogiso, H. Tokumoto, and U. Dürig, Appl. Phys. Lett. 75, 3132 (1999).

${ }^{95}$ S. B. Legoas, V. Rodrigues, D. Ugarte, and D. S. Galvão, Phys. Rev. Lett. 93, 216103 (2004).

${ }^{96}$ S. B. Legoas, V. Rodrigues, D. Ugarte, and D. S. Galvão, Phys. Rev. Lett. 95, 169602 (2005). 